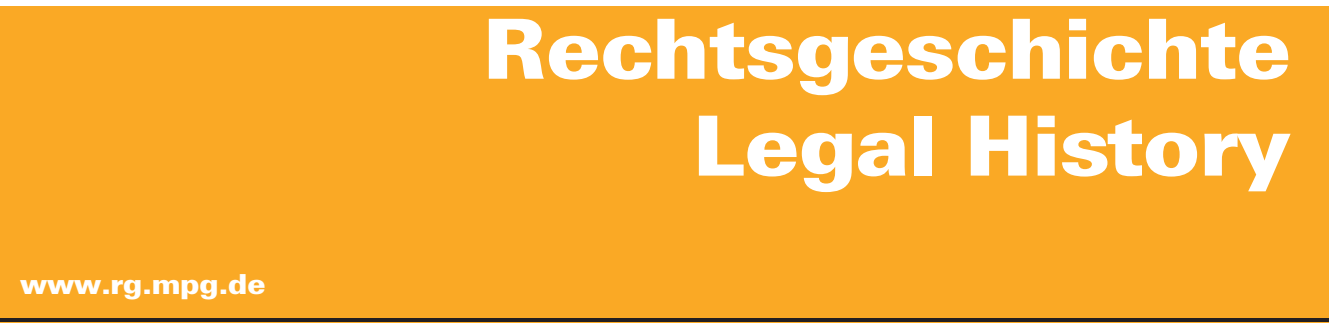

http://www.rg-rechtsgeschichte.de/rg23

Rg 2015

$274-276$

Zitiervorschlag: Rechtsgeschichte - Legal History Rg 23 (2015)

http://dx.doi.org/10.12946/rg23/274-276

\title{
Sandro Wiggerich
}

\section{Rechtsgeschichte und Bedeutung}




\section{Sandro Wiggerich Rechtsgeschichte und Bedeutung}

Alle Rechtsgeschichte ist Normengeschichte. Dies folgt aus der Definition einer Wissenschaft durch Methode und Gegenstand: "wo mit neuer Methode einem neuen Problem nachgegangen wird und dadurch Wahrheiten entdeckt werden, welche neue bedeutsame Gesichtspunkte eröffnen, da entsteht eine neue $>$ Wissenschaft $\ll{ }^{1}{ }^{1}$ Die Rechtsgeschichte bedient sich - darin ist sie Geschichtswissenschaft - historischer Methoden; ihr Proprium ist es, diese auf den Gegenstand »Recht « anzuwenden. Ungeachtet der Schwierigkeiten, sich auf einen Begriff des Rechts schlechthin oder auch nur für eine bestimmte Zeit zu verständigen, dürfte weitgehende Einigkeit darüber bestehen, dass Recht jedenfalls (qualifizierte) Normen voraussetzt. Diese Normbezogenheit konstituiert damit die Rechtsgeschichte als Fach und grenzt sie von anderen Teilbereichen der Geschichtswissenschaft $\mathrm{ab}$.

Diesen Zusammenhang scheint Peter Oestmann in seiner sehr persönlich geprägten Stellungnahme zunächst zu bestreiten. Für ihn ist Normengeschichte nur eine von mindestens drei Arten, Rechtsgeschichte zu betreiben. Neben diese treten gleichrangig die Wissenschaftsgeschichte und die Geschichte der Rechtspraxis. Im Koordinatensystem des Faches eröffnet er damit eine weitere Dimension, die quer zu den traditionellen Einteilungen $^{2}$ nach (historisch-nationalen) Rechtsmassen, Epochen und geographischen Räumen liegt. Während deren jeweils maßgebliches Kriterium offen hervortritt, bleibt die für Oestmann leitende Kategorie jedoch diffus. Er selbst bezieht sich ausdrücklich auf »methodisch-handwerkliche Gesichtspunkte « und spricht die Bedeutung verschiedener Quellengruppen an. Die drei Zugänge zur Rechtsgeschichte beschreibt er dann zunächst nach ihren typischen Fragestellungen, bevor er ihnen eine Quellengattung zuordnet. Bereits hier wird deutlich, dass die Quellenauswahl eher ein
Reflex der Forschungsfrage ist. Beide stehen jedoch nicht in einem notwendigen Bedingungszusammenhang: Die Erkenntnis einer Observanz aus Gerichtsakten beantwortet gerade die Frage nach dem Inhalt einer Rechtsnorm. Ebenso erlauben Papyri den Schluss auf »Hintergründe und Gestalt « von Rechtsanschauungen, ${ }^{3}$ also Norminhalte. Umgekehrt spiegeln der Erlass und die Auslegung von Rechtsnormen auch Probleme und Erfordernisse der Rechtspraxis. Zudem bleiben wichtige Quellengruppen - insbesondere Sach-, aber auch narrative Quellen - bei der Einteilung außen vor.

Die Flüchtigkeit der Unterscheidung erkennt auch Oestmann, lässt die erforderlichen Korrekturen jedoch weitgehend offen. Betrachtet man die von ihm skizzierten Zugänge, scheint ihr Kriterium weniger eine bestimmte Quellengattung als vielmehr der ihnen innewohnende Bedeutungsmaßstab zu sein. Die Eigenheit der Normengeschichte in dem engeren Sinne einer Dogmenoder Problemgeschichte liegt nicht darin, sich mit Normen zu befassen. Ihr Wesen ist es, die Gedanken "großer Juristen « zu erforschen; sie ist die intellektuelle »Biographie großer Männer ${ }^{4}{ }^{4}$ auf dem Gebiet des Rechts. Maßstab dieser angenommenen Größe ist die Wirkungsgeschichte. Bedeutung kommt einer Äußerung hiernach vor allem dann zu, wenn sie in ihrer Zeit und über die Zeiten hinweg das Denken anderer befruchtet und mitunter Spuren im geltenden Recht hinterlassen hat. Am stärksten gilt dies für die Äußerungen des Gesetzgebers selbst. Dagegen befasst sich die Praxisgeschichte mit den Scharen derjenigen Juristinnen und Juristen, die nicht über ihren Lebenskreis hinaus gewirkt haben. Deren Bedeutung für die Gesellschaft ihrer Zeitgenossen muss dabei nicht notwendig geringer gewesen sein. Für Helena Curtens, die 1738 als Hexe zum Tode verurteilt wurde, ${ }^{5}$ waren die Rechtsansichten eines ansonsten wenig bekannten Amtsrichters in Gerresheim

1 WeBer (1922) 166.

2 Knapper Überblick bei STOLLEIS (2007).

3 WolfF (2002) 1 und passim.

4 Carlyle (1841) 47; Übers. d. Verf.

5 Einen Überblick über den Fall gibt MÜNSTER-SCHRÖER (2009). 
viel bedeutender als die gegenteilige und historisch wirkmächtigere Auffassung eines Christian Thomasius.

Dogmen- und Praxisgeschichte spiegeln damit im Kleinen die Unterschiede von Politischer und Alltagsgeschichte. Die unterschiedlichen, unausgesprochenen Bedeutungsmaßstäbe sind es, die die unterschiedlichen Zugänge zur Rechtsgeschichte prägen. Aus ihnen folgt zwingend die Auswahl einer bestimmten Gruppe von Akteuren und damit schließlich auch typischer Quellen. Die Frage danach, auf welche Akteure eine rechtshistorische Untersuchung abzielt, markiert damit die eigentliche Grenze zwischen Dogmen- und Praxisgeschichte. Einige Juristen auf der Grenze von Theorie und Praxis werden durch diese Unterscheidung gleichsam entzweigeschnitten. David Mevius oder Georg Arnold Heise erlangten ihre Bedeutung am Maßstab der Dogmengeschichte durch den intellektuellen Einfluss ihrer Schriften. Ihre Arbeitsweise und die Genese ihrer Entscheidungen im konkreten Einzelfall zu ergründen, verbleibt der Geschichte der Rechtspraxis. ${ }^{6}$

Eine seltsame Zwischenstellung nehmen quantifizierende Ansätze ein. Wer in sozialhistorischer Absicht Gerichtsakten seriell auswertet, ${ }^{7}$ schöpt zwar aus der Rechtspraxis, misst aber dem Einzelfall gerade keine besondere Bedeutung bei. Eine juristische Auffassung wäre hiernach historisch vor allem dann bedeutsam, wenn sie von möglichst vielen geteilt wird. Wie vieler Amtsrichter bedarf es aber, um einen Professor aufzuwiegen? Ob ein Argument in einem oder in zehn Prozent aller bekannten Anwendungsfälle gebraucht wurde, sagt wenig über seine Qualität aus. Wenn man den Satz Marie Theres Fögens vom Recht als Kommunikation über Recht und Unrecht ${ }^{\mathbf{8}}$ ernst nimmt, muss man zur historischen Erforschung des Rechts vielmehr die Argumente jedes Akteurs für sich würdigen. Die Bedeutung eines Gedankens ist in dem Moment, in dem er in die Welt tritt, noch völlig unklar; er gewinnt sie erst durch äußere Zuschreibungen. Die Einzelmeinung eines Professors kann schon bei seinem Tod vergessen sein, das Urteil eines Richters nur bis zur Entscheidung der nächsten Instanz Bestand haben.
Aus einem solchen diskursiven Verständnis von Recht folgt die Gleichrangigkeit von Dogmen- und Praxisgeschichte. Wegen der bloß subjektiven Bedeutungszuschreibung fehlt ein objektiver Maßstab, der ein Vorrangverhältnis zwischen einzelnen Argumenten begründen könnte. Subsumtion ist gerade keine historische Methode. Es lässt sich lediglich mit der Methodengeschichte fragen, ob eine Äußerung nach den Diskursregeln ihrer Zeit Anerkennung als juristisches Argument beanspruchen konnte. ${ }^{9}$ Damit wird zugleich der Sinn einer bloß vergleichenden Gegenüberstellung von Lehre und Praxis zweifelhaft. Reizvoller als ein solcher Vergleich ist es, Diskurszusammenhänge zwischen einzelnen Akteuren in Theorie und Praxis aufzuzeigen. Hier hat auch die Art von Wissenschaftsgeschichte ihren Platz, die einzelne Autoren in ihren Denktraditionen und Stilrichtungen untersucht.

Umschreibt man den Inhalt des Faches in dieser Weise, folgt daraus, dass nicht alles, was Rechtshistoriker tun, notwendig auch Rechtsgeschichte ist. Für die Disziplingeschichte der Rechtsgeschichte selbst tritt dies entgegen der Auffassung Oestmanns schon deshalb offen zutage, weil diese gar nicht nach Recht und Unrecht fragt. Sie ist als Fach vielmehr Teil der Wissenschafts- und hier der Historiographiegeschichte. In abgeschwächtem Maße gilt dies auch für die »äußere« Geschichte einzelner juristischer Institutionen oder Berufsgruppen, insbesondere die Wissenschaftsgeschichte des Universitätsbetriebes. Dieser soziale Kontext erlangt rechtshistorische Bedeutung nur insoweit, wie er als Schaffensrahmen auf den Normdiskurs eingewirkt hat. Auch die Erforschung juristischer Sachund Bildquellen ist zunächst spezialisierte Archäologie und Ikonographie, also Hilfswissenschaft. ${ }^{10}$ Erst im Schluss von diesen Quellen auf Rechtsinhalte vollzieht sich rechtshistorische Forschung im eigentlichen Sinne.

Was aber leistet die Einteilung rechtshistorischer Zugänge nach ihren impliziten Bedeutungsmaßstäben über die Möglichkeit einer Verortung hinaus? Einen Ausbruch aus der von Oestmann beklagten Froschperspektive garantiert sie nicht. Thematische Engführungen ergeben sich aus dem
6 Dogmengeschichtliche Perspektive auf Mevius' Werk etwa bei Wurch (2015); die Grenze zwischen »Normen-« und Praxisgeschichte aufzulösen versucht Тном⿰сн (2014).
7 Stodolkowitz (2011); klassisch RANIERI (1985).

8 Fögen (2002) 15, Ziff. 6.

9 Zu den Wandlungen SCHröder (2012).
10 Anders Lück (2012) 37.

Sandro Wiggerich 
Maß, nicht aus der Art der Eingrenzung. Die Offenlegung des eigenen Bedeutungsmaßstabs und die Frage nach den relevanten historischen Akteuren können aber dazu dienen, Forschungsvorhaben zu strukturieren und Anknüpfungspunkte $\mathrm{zu}$ identifizieren. Zudem bieten diese Aspekte einen Deutungsansatz für die Schärfe, mit der Methodendebatten bisweilen geführt werden: Wer einen Vorrang des eigenen rechtshistorischen Ansatzes behauptet, wirft damit nur vordergründig eine Sachfrage auf. Implizit wertet er auch den Bedeutungsmaßstab des anderen ab. Wegen der unentrinnbaren Subjektivität der Zuschreibung rechtshistorischer Bedeutung liegt darin immer zugleich ein persönlicher Angriff. Wissenschaftstheoretische Überlegungen vermögen vielleicht nicht, die letztlich nur soziologisch zu erfassende Kluft zwischen den verschiedenen Denktraditionen der heutigen Vertreter des Faches zuzuschütten. Die Erkenntnis, aus getrennten Richtungen an einem gemeinsamen Werk zu bauen, gemahnt jedoch zu einer gelassenen Haltung.

\section{Bibliographie}

- Carlyle, Thomas (1841), On Heroes, Hero-Worship and the Heroic in History, London

- Fögen, Marie Theres (2002), Rechtsgeschichte - Geschichte der Evolution eines sozialen Systems, in: Rg. Rechtsgeschichte 1, $14-20$

- Lück, Heiner (2012), Was ist und was kann Rechtsarchäologie?, in: Denkströme. Journal der Sächsischen Akademie der Wissenschaften, Heft 8/2012, 35-55

- Münster-Schröer, Erika (2009), Hexenverfolgungen in Düsseldorf, in: Lexikon zur Geschichte der Hexenverfolgung, hrsg. v. Gudrun Gersmann, Katrin Moeller und Jürgen-Michael Schmidt, https://www.historicum.net/purl/ffzoy/ (abgerufen am 20.03.2015)

- Ranieri, Filippo (1985), Recht und Gesellschaft im Zeitalter der Rezeption. Eine rechts- und sozialgeschichtliche Analyse der Tätigkeit des Reichskammergerichts im 16. Jahrhundert, Köln

- Schröder, Jan (2012), Recht als Wissenschaft. Geschichte der juristischen Methodenlehre in der Neuzeit (1500-1933), 2. Aufl., München

- Stodolkowitz, Stefan Andreas (2011), Das Oberappellationsgericht Celle und seine Rechtsprechung im 18. Jahrhundert, Köln

- Stolleis, Michael (2007), Rechtsgeschichte, Verfassungsgeschichte, in: Goertz, Hans-Jürgen (Hg.), Geschichte. Ein Grundkurs, 3. Aufl., Hamburg, 391-412

- Thomsch, Astrid (2014), David Mevius und der (Prozess-)Vergleich im Usus modernus pandectarum. Eine Analyse von Gerichtsordnung, Decisionen und Akten, Hamburg

- Weber, Max (1922), Die »Objektivität« sozialwissenschaftlicher und sozialpolitischer Erkenntnis (1904), in: DERs., Gesammelte Aufsätze zur Wissenschaftslehre, Tübingen, 146-214

- Wolff, Hans Julius (2002), Das Recht der griechischen Papyri Ägyptens in der Zeit der Ptolemaeer und des Prinzipats, 1. Band, München

- Wurch, Nils (2015), David Mevius und das lübische Recht. Dargestellt am Beispiel des »beneficium excussionis«, Köln 